BMJ Open

Diabetes

Research

\& Care

\section{Insulin-sensitive and insulin-resistant obese and non-obese phenotypes: role in prediction of incident pre-diabetes in a longitudinal biracial cohort}

\author{
Ibiye Owei, ${ }^{1}$ Nkiru Umekwe, ${ }^{1}$ Casey Provo, ${ }^{1}$ Jim Wan, ${ }^{2}$ Samuel Dagogo-Jack ${ }^{1}$
}

To cite: Owei I, Umekwe N, Provo C, et al. Insulin-sensitive and insulin-resistant obese and non-obese phenotypes: role in prediction of incident pre-diabetes in a longitudinal biracial cohort. BMJ Open Diab Res Care 2017;5:e000415. doi:10.1136/ bmjdrc-2017-000415

Received 12 March 2017 Revised 25 April 2017 Accepted 11 June 2017

\section{(D) CrossMark}

${ }^{1}$ Division of Endocrinology, Diabetes and Metabolism, University of Tennessee Health Science Center, Memphis, Tennessee, USA ${ }^{2}$ Department of Preventive Medicine, University of Tennessee Health Science Center, Memphis, Tennessee, USA

Correspondence to Dr Samuel Dagogo-Jack; sdj@uthsc.edu

\section{ABSTRACT}

Objective We measured insulin sensitivity with euglycemic clamp (Si-clamp) in initially normoglycemic African Americans (AA) and European Americans (EA), to probe the existence of subphenotypes of obesity and leanness, and their impact on incident dysglycemia during longitudinal follow-up.

Research design and methods 320 healthy subjects (176 AA, $144 \mathrm{EA}$; mean age 44.2 \pm 10.6 years) underwent baseline assessments, including Si-clamp and homeostasis model of insulin resistance (HOMA-IR) and were stratified into: insulin-resistant obese (IRO) (body mass index (BMI) $\geq 30 \mathrm{~kg} / \mathrm{m}^{2}$, Si-clamp $<0.1$, HOMA-IR $\geq 2.5$ ); insulinsensitive obesity (ISO) (BMI $\geq 30 \mathrm{~kg} / \mathrm{m}^{2}$, Si-clamp $\geq 0.1$, HOMA-IR <2.5); insulin-resistant non-obese (IRN) (BMI $<28 \mathrm{~kg} / \mathrm{m}^{2}$, Si-clamp $<0.1$, HOMA-IR $\geq 2.5$ ); insulinsensitive non-obese (ISN) (BMI $<28 \mathrm{~kg} / \mathrm{m}^{2}$, Si-clamp $\geq 0.1$, HOMA-IR <2.5). Outcome measures were cardiometabolic risks and incident pre-diabetes/type 2 diabetes (T2D) during 5.5 years.

Results Compared with IRO, subjects with ISO had lower abdominal fat, triglycerides and high-sensitivity $\mathrm{C}$ reactive protein and higher adiponectin $(p=0.015$ to $<0.0001)$. IRN subjects had higher cardiometabolic risk markers than ISN ( $p=0.03$ to $<0.0001$ ). During 5.5-year follow-up, incident pre-diabetes/T2D was lower in ISO (31.3\% vs $48.7 \%$ ) among obese subjects and higher in IRN $(47.1 \%$ vs. $26.0 \%$ ) among non-obese subjects $(\mathrm{p}=0.0024)$. KaplanMeier analysis showed significantly different pre-diabetes/ T2D survival probabilities across insulin sensitivity/ adiposity phenotypes $(p=0.0001)$.

Conclusions Insulin sensitivity predicts $\sim 40 \%$ decrease in the relative risk of incident pre-diabetes/T2D among obese persons, whereas insulin resistance predicts $~ 80 \%$ increased risk among non-obese persons. This is the first documentation of healthy and unhealthy phenotypes of obesity and leanness in a prospective biracial cohort, using rigorous measurement of insulin sensitivity.

\section{INTRODUCTION}

A phenotype of obesity characterized by preserved insulin sensitivity and paucity of components of the metabolic syndrome is being increasingly recognized. ${ }^{1-5}$ Variously referred to as 'metabolically healthy obesity', 'metabolically benign obesity', or

\section{Significance of this study}

What is already known about this subject?

- Previous studies have identified subsets of metabolically healthy obese (MHO) and metabolically obese, normal weight (MONW) individuals.

- Although implied as the underlying mechanism for the MHO and MONW phenotypes, insulin sensitivity is seldom measured directly in the published reports.

What are the new findings?

- Using hyperinsulinemic euglycemic clamp to directly assess insulin sensitivity in a normoglycemic cohort, the present study documents insulin-sensitive and insulin-resistant phenotypes among obese and nonobese African Americans and European Americans.

- Insulin-sensitive obese (ISO) subjects had a favorable cardiometabolic risk profile and showed decreased risk of incident dysglycemia, compared with insulinresistant obesity.

- Insulin-resistant non-obese (IRN) subjects exhibited an unfavorable cardiometabolic risk profile and accelerated progression from normoglycemia to dysglycemia during 5.5 years of follow-up.

- The observed ISO and IRN phenotypes roughly mirror the MHO and MONW characteristics.

How might these results change the focus of research or clinical practice?

- A longer follow-up study is needed to assess the stability of the ISO phenotype and to determine whether the association between insulin-sensitivity phenotypes and incident pre-diabetes extends to type 2 diabetes.

- Intervention studies are needed to determine the impact of insulin-sensitivity phenotypes on the efficacy of lifestyle interventions on prevention of type 2 diabetes and reversal of pre-diabetes.

'insulin-sensitive obesity' (ISO), this phenotype has been reported in approximately $10 \%-40 \%$ of obese people,${ }^{6-9}$ including those with extreme obesity. ${ }^{8}$ The obverse-a subset of normal weight persons who harbor $\geq 2$ components of the metabolic syndromehas also been described. ${ }^{8-10}$ Such individuals 
have been referred to as having the "metabolically obese, normal weight' (MONW) phenotype. ${ }^{8-10}$ As conventionally defined, metabolically healthy obesity exists in any individual who is obese (body mass index (BMI) $\geq 30 \mathrm{~kg} /$ $\mathrm{m}^{2}$ ) but has fewer than two components of the metabolic syndrome and shows evidence of preservation of insulin sensitivity. ${ }^{1-10}$ The latter is usually documented by calculating the homeostasis model of insulin resistance (HOMA-IR) from fasting insulin and glucose levels or indices derived from oral glucose tolerance tests. ${ }^{1-10}$ Fasting glucose, waist circumference, blood pressure (BP), triglycerides and high-density lipoprotein (HDL) cholesterol levels are among the five metabolic syndrome components ${ }^{11}$ used to classify individuals as metabolically 'healthy' or 'unhealthy'. ${ }^{1-10}$ However, these are not mutually exclusive traits, as much collinearity exists among the components. ${ }^{12}{ }^{13}$ Moreover, some of the metabolic syndrome markers (such as triglycerides and HDL cholesterol) display ethnic variations in normative values. ${ }^{14}$

As insulin resistance drives metabolic syndrome components in unfavorable directions,${ }^{15}$ the use of those same components for defining phenotypes of obesity is a metaphor for documentation of insulin sensitivity. Publications describing the metabolically healthy or unhealthy phenotypes of obesity and leanness have traditionally been based on less rigorous assessment of insulin sensitivity, despite the implied centrality of insulin action in the classification. ${ }^{16}$ However, more recent studies in the field have used hyperinsulinemic euglycemic clamp to define insulin sensitivity status. ${ }^{17-19}$ Numerous studies have reported that the metabolically healthy obese (MHO) phenotype may be associated with a better cardiometabolic risk profile and decreased incidence of type 2 diabetes (T2D) compared with the metabolically unhealthy phenotype. ${ }^{1-8} 16^{172021}$ In the Framingham Offspring Study of predominantly white subjects, insulin sensitivity status (as determined by HOMA-IR) significantly influenced the risk of incident T2D, with lower hazard among insulin-sensitive obese subjects and higher hazard among insulin-resistant non-obese (IRN) subjects. ${ }^{20}$ In a pooled analysis of eight prospective studies, the adjusted relative risk for incident T2DM was 2.2-fold higher in metabolically unhealthy obese subjects compared with MHO subjects. ${ }^{21}$

Clearly, the concept of metabolically healthy and unhealthy phenotypes of obesity and lean stature remains intriguing. However, data in the literature are limited by imprecise definitions, restricted demography, and the use of surrogates or superficial assessment of insulin sensitivity. ${ }^{16}$ Another drawback is inclusion of elevated fasting glucose among the risk markers used for defining the phenotypes: even mild elevations in plasma glucose can alter lipid metabolism and activate reactive oxygen species and proinflammatory cytokines, making it difficult to separate primary from secondary phenomena. ${ }^{22}{ }^{23}$ The Pathobiology of Pre-diabetes in a Biracial Cohort (POP$\mathrm{ABC}$ ), a longitudinal study of initially normoglycemic
African Americans (AA) and European Americans (EA) with parental T2D, ${ }^{24-26}$ afforded an opportunity to use rigorously defined insulin sensitivity status to probe the nosological phenotypes of obese and non-obese habitus, denominated by heterogeneity in insulin sensitivity, in a biracial cohort. Specifically, we aimed to answer the following questions: (1) to what extent do similarly obese individuals differ regarding hepatic and wholebody insulin sensitivity; (2) to what extent do similarly non-obese individuals differ regarding hepatic and whole-body insulin sensitivity; and (3) to what extent does the presence of insulin sensitivity in obese subjects, or insulin resistance in non-obese subjects, affect the rate of progression from normoglycemia to pre-diabetes/ T2D?

\section{RESEARCH DESIGN AND METHODS \\ Study subjects}

The study subjects were participants in the POP-ABC study. ${ }^{24-26}$ Inclusion criteria for the POP-ABC study were as follows: age 18-65 years; non-Hispanic white (EA) or non-Hispanic black (AA) race/ethnicity status; biological parent(s) with T2D. In addition, participants completed a screening of $75 \mathrm{~g}$ oral glucose tolerance test (OGTT) and were enrolled if they had normal fasting plasma glucose (FPG) $(<100 \mathrm{mg} / \mathrm{dL}(5.6 \mathrm{mmol} / \mathrm{L}))$ or normal glucose tolerance (2-hour plasma glucose $(2 \mathrm{hrPG})<140 \mathrm{mg} / \mathrm{dL}$ $(7.8 \mathrm{mmol} / \mathrm{L}))$. All participants were ambulatory, in good overall health, and were not taking medications known to alter body weight, insulin sensitivity, glucose, lipids or BP. The University of Tennessee Institutional Review Board approved the study and all participants signed written informed consent before initiation of study, which was conducted according to the World Medical Association's Declaration of Helsinki.

\section{Assessments}

All procedures were conducted at the University of Tennessee General Clinical Research Center (GCRC). Baseline assessments included medical history and examination, measurement of height, weight and waist circumference, and a standard $75 \mathrm{~g}$ OGTT. ${ }^{26}$ The BMI was calculated as the weight in kilogram divided by the height in meter squared. Measures of food habits and physical activity were also recorded, as previously described. ${ }^{27}$

Total, trunk, and lean body mass were measured using DEXA. Additional assessments included FPG measured quarterly, OGTT annually, insulin secretion annually, and insulin sensitivity in years 1,3 and 5 , as previously described. ${ }^{24-26}$ The primary outcome was the development of pre-diabetes (FPG 100-125 mg/dL (5.6-6.9 mmol/L) and/or $2 \mathrm{hrPG} 140-199 \mathrm{mg} / \mathrm{dL}(7.8-11.0 \mathrm{mmol} / \mathrm{L}))$ or diabetes, as defined by the American Diabetes Association criteria. 28

\section{Insulin sensitivity and insulin secretion}

Whole-body insulin sensitivity was measured using the hyperinsulinemic euglycemic clamp method of 
DeFronzo et $a l^{30}$ In brief, subjects who had fasted overnight underwent the clamp studies at the GCRC. After placement of intravenous cannulas in both arms, a primed, continuous intravenous infusion of regular insulin $(2 \mathrm{mU} / \mathrm{kg} / \mathrm{min} ; 14.4 \mathrm{pmol} / \mathrm{kg} / \mathrm{min})$ was administered for $180 \mathrm{~min}$ while maintaining blood glucose level at $\sim 100 \mathrm{mg} / \mathrm{dL}(5.6 \mathrm{mmol} / \mathrm{L})$ with a variable rate dextrose (20\%) infusion. Arterialized blood sampling was performed every $10 \mathrm{~min}$. The rate of total insulin-stimulated glucose disposal (M) was calculated from the dextrose infusion rate during steady state (final $60 \mathrm{~min}$ of insulin infusion). The $\mathrm{M}$ value was corrected for the steady-state plasma insulin levels, to derive the final insulin sensitivity index (Si-clamp, $\mu \mathrm{mol} / \mathrm{kg}$ fat-free mass/ $\mathrm{min} / \mathrm{pmol} / \mathrm{L}$ ). ${ }^{24} 262930$ Acute insulin response to glucose (AIR) was assessed using the frequently sampled intravenous glucose tolerance test, as previously described. ${ }^{24} 26$ The HOMA-IR, an estimate of hepatic insulin sensitivity, and (HOMA-B), an estimate of beta-cell function, were derived from fasting glucose and insulin values. ${ }^{31}$

\section{Biochemical measurements}

Plasma glucose was measured with a glucose oxidase method (Yellow Spring Instruments, Yellow Spring, $\mathrm{OH}$, USA). Plasma levels of insulin, high-sensitivity $\mathrm{C}$ reactive protein (hsCRP), adiponectin and leptin were measured with commercial ELISA kits in our Endocrine Research Laboratory. Hemoglobin A1c (HbA1c) and fasting plasma lipid profiles were measured in a contract clinical laboratory.
Definition of insulin sensitivity and adiposity subgroups

Using BMI data from our cohort, we stratified study subjects into obese (BMI $\geq 30 \mathrm{~kg} / \mathrm{m}^{2}$ ) and non-obese $\left(\right.$ BMI $\left.<28 \mathrm{~kg} / \mathrm{m}^{2}\right)$ groups. From the cohort distributions of Si-clamp and HOMA-IR values, we defined insulin resistance as a Si-clamp value below the 25th percentile $(<1.0 \mu \mathrm{mol} / \mathrm{kg}$ fat-free mass $/ \mathrm{min} / \mathrm{pmol} / \mathrm{L})$ and $/$ or an HOMA-IR value above the 75 th percentile $(\geq 2.5)$. Thus, the BMI and insulin sensitivity data enabled the stratification of study subjects into four subgroups: (1) insulin-resistant obese (IRO) (BMI $\geq 30 \mathrm{~kg}$ / $\mathrm{m}^{2}$, Si-clamp <0.1 and/or HOMA-IR $\geq 2.5$ ); (2) ISO (BMI $\geq 30 \mathrm{~kg} / \mathrm{m}^{2}$, Si-clamp $\geq 0.1$ and/or HOMA-IR $<2.5$; (3) IRN (BMI $<28 \mathrm{~kg} / \mathrm{m}^{2}$, Si-clamp $<0.1$ and/or HOMA-IR $\geq 2.5$ ); (4) insulin-sensitive non-obese (ISN) $\left(\mathrm{BMI}<28 \mathrm{~kg} / \mathrm{m}^{2}\right.$ and $/$ or Si-clamp $\geq 0.1$, HOMA-IR $<2.5$ ). Figure 1 shows the regression plots of Si-clamps and HOMA-IR versus BMI, the agreement between the two measures of insulin sensitivity, and the percentile distribution of Si-clamp and HOMA-IR.

\section{Statistical analysis}

Data were reported as means \pm SD. Significance level was set as $\mathrm{p}<0.05$. Percentile plots were generated to determine the 25th and 75th percentiles for Si-clamp and HOMA-IR, respectively. Unpaired t-tests were used to compare continuous variables and $\chi^{2}$ and $\chi^{2}$ for trend (Cochran-Armitage) tests were used for categorical data. General linear regression models were used to compare baseline anthropometric and cardiometabolic
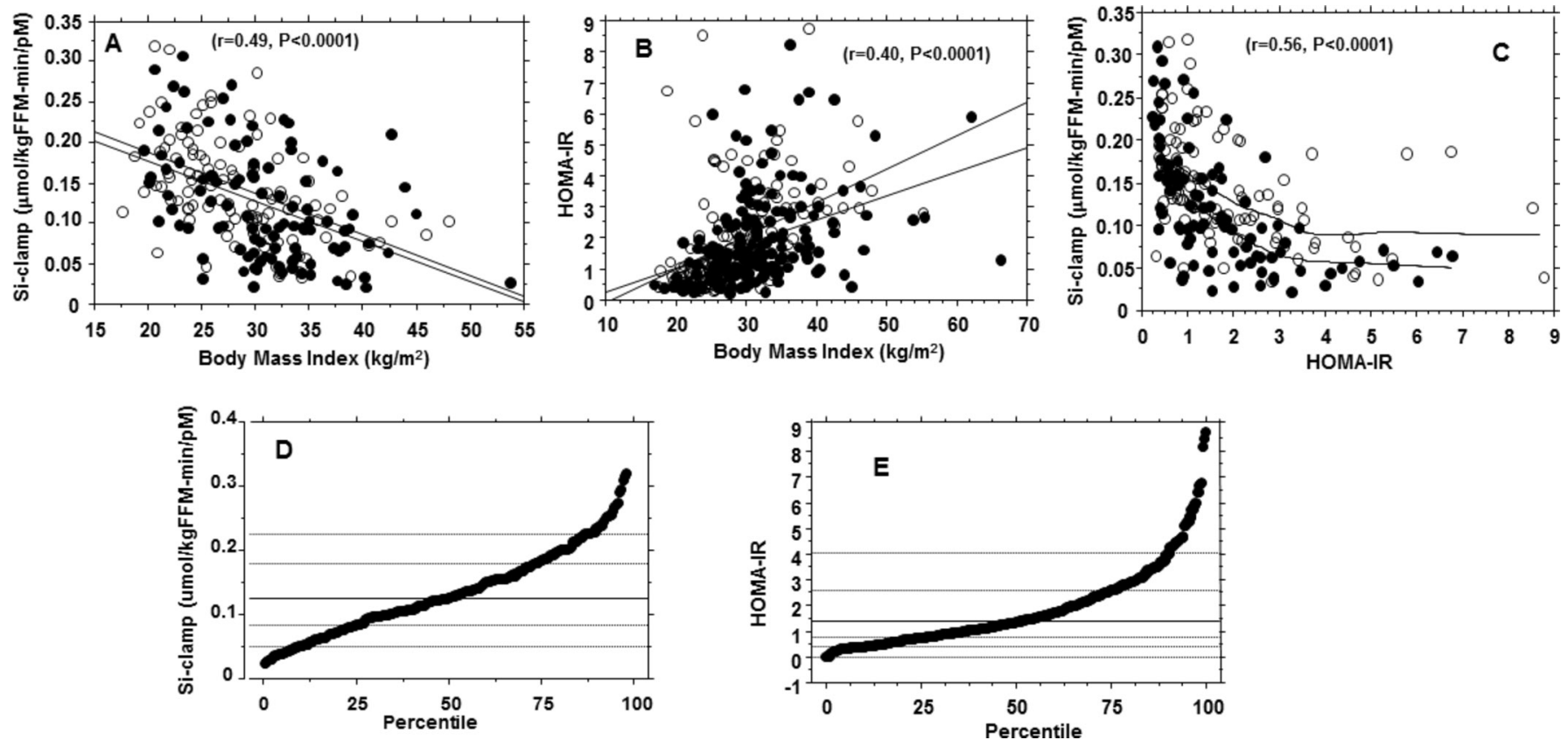

Figure 1 Upper panel: Regression plots of insulin sensitivity (Si-clamp) (A) and homeostasis model of insulin resistance (HOMA-IR) (B) versus body mass index, and Si-clamp versus HOMA-IR (C), in African Americans (closed circles) and European Americans (open circles). Lower panel: Percentile distribution of Si-clamp (D) and HOMA-IR (E) in the study cohort. HOMA-IR data were obtained in 320 subjects, of whom 206 underwent hyperinsulinemic euglycemic clamp to generate Si-clamp data. 
Table 1 Baseline clinical and biochemical characteristics of study subjects by obesity and insulin sensitivity status

\begin{tabular}{lllllll}
\hline Characteristic & IRO & ISO & p Value & IRN & ISN & p Value \\
\hline Number & 76 & 96 & & 17 & 131 & \\
\hline Gender (F/M) & $58 / 18$ & $70 / 26$ & 0.64 & $9 / 8$ & $91 / 40$ & 0.17 \\
\hline Ethnicity (AA/EA) & $51 / 25$ & $58 / 38$ & 0.39 & $8 / 9$ & $72 / 59$ & 0.54 \\
\hline Age (year) & $44.0 \pm 12.1$ & $44.0 \pm 10.6$ & 0.97 & $42.5 \pm 7.00$ & $46.2 \pm 9.42$ & 0.11 \\
\hline BMI (kg/m ${ }^{2}$ ) & $36.7 \pm 6.86$ & $35.1 \pm 5.26$ & 0.04 & $26.4 \pm 2.23$ & $24.2 \pm 2.89$ & 0.003 \\
\hline HOMA-IR & $3.51 \pm 1.60$ & $1.49 \pm 0.83$ & $<0.0001$ & $3.35 \pm 1.58$ & $1.09 \pm 0.10$ & $<0.0001$ \\
\hline Si-clamp & $0.058 \pm 0.025$ & $0.138 \pm 0.048$ & $<0.0001$ & $0.065 \pm 0.019$ & $0.176 \pm 0.056$ & $<0.0001$ \\
\hline FPG (mg/dL) & $93.3 \pm 6.47$ & $92.7 \pm 7.57$ & 0.60 & $93.6 \pm 5.78$ & $90.7 \pm 6.29$ & 0.08 \\
\hline 2hrPG (mg/dL) & $128 \pm 27.2$ & $125 \pm 24.2$ & 0.42 & $129 \pm 23.8$ & $121 \pm 26.8$ & 0.19 \\
\hline HbA1c (\%) & $5.59 \pm 0.56$ & $5.64 \pm 0.44$ & 0.24 & $5.58 \pm 0.42$ & $5.51 \pm 0.40$ & 0.22 \\
\hline Food habits score & $2.64 \pm 0.50$ & $2.57 \pm 0.44$ & 0.36 & $2.70 \pm 0.46$ & $2.54 \pm 0.55$ & 0.26 \\
\hline Physical activity (Met-hour/week) & $18.0 \pm 42.2$ & $13.8 \pm 19.6$ & 0.43 & $13.7 \pm 21.3$ & $22.5 \pm 35.1$ & 0.32 \\
\hline
\end{tabular}

To convert the values for glucose to millimoles per liter, multiply by 0.0555 .

2hrPG, 2-hour plasma glucose during $75 \mathrm{~g}$ oral glucose tolerance test; AA, African American; EA, European American; FPG, fasting plasma glucose; HbA1c, hemoglobin A1c; HOMA-IR, homeostasis model of insulin resistance; IRN, insulin-resistant non-obese; IRO, insulinresistant obese; ISN, insulin-sensitive non-obese; ISO, insulin-sensitive obese; Si-clamp, insulin sensitivity by hyperinsulinemic euglycemic clamp expressed as $\mu \mathrm{mol} / \mathrm{kg}$ fat-free $\mathrm{mass} / \mathrm{min} / \mathrm{pmol} / \mathrm{L}$.

characteristics in IRO, IRN, ISO and ISN groups of subjects. The relationship between Si-clamp or HOMA-IR versus BMI was analyzed using linear regression and Pearson correlation coefficients. The relationship between Si-clamp and HOMA-IR was analyzed using polynomial regression with Lowess model for curve fitting. Kaplan-Meier plots for the probability of incident pre-diabetes/diabetes in defined groups of obese and non-obese subjects were compared using log-rank test. Statistical analyses were performed with Statview and SAS statistical software, V.9.3 (SAS Institute).

\section{RESULTS}

\section{Cohort characteristics}

We analyzed data from 320 (176 AA, $144 \mathrm{EA})$ study participants. At enrollment, the mean $( \pm \mathrm{SD})$ age was $44.2 \pm 10.6$ years, BMI was $30.2 \pm 7.23 \mathrm{~kg} / \mathrm{m}^{2}$, FPG was $91.8 \pm 6.77 \mathrm{mg}$ / $\mathrm{dL}, 2 \mathrm{hrPG}$ was $124 \pm 25.8 \mathrm{mg} / \mathrm{dL}$, and $\mathrm{HbAlc}$ was $5.56 \% \pm 0.44 \%$, for the cohort. The mean systolic BP was $121 \pm 16.1 \mathrm{~mm} \mathrm{Hg}$, diastolic BP was $72.8 \pm 9.1 \mathrm{~mm} \mathrm{Hg}$, triglycerides was $94.3 \pm 53.3 \mathrm{mg} / \mathrm{dL}$, HDL cholesterol was $52.6 \pm 13.7 \mathrm{mg} / \mathrm{dL} \quad(46.1 \pm 12.4 \mathrm{mg} / \mathrm{dL}$ in men; $55.2 \pm 13.4 \mathrm{mg} / \mathrm{dL}$ in women) and waist circumference was $94.3 \pm 15.6 \mathrm{~cm}(97.9 \pm 15.2 \mathrm{~cm}$ in men; $92.7 \pm 15.6 \mathrm{~cm}$ in women). Table 1 shows the baseline characteristics for participants in the four insulin sensitivity/adiposity strata.

Subjects in the ISN group were, on average, 2-4 years older than those in the other groups, but the age differences were not statistically significant. Similarly, there were no significant differences in gender or ethnic composition across the subgroups. Glycemic measures (FPG, 2hrPG and HbAlc) at enrollment were similar across subgroups, as expected in a cohort selected for initial normoglycemia. ${ }^{24-26}$ The mean BMI values in the two obese subgroups $\left(36.7 \mathrm{~kg} / \mathrm{m}^{2}\right.$ in IRO and $35.1 \mathrm{~kg} / \mathrm{m}^{2}$ in ISO) and the two non-obese subgroups $\left(26.4 \mathrm{~kg} / \mathrm{m}^{2}\right.$ in IRN and $24.2 \mathrm{~kg} / \mathrm{m}^{2}$ in ISN) were in line with the study targets. Self-reported food habits and physical activity were not significantly different across the subgroups (table 1).

The HOMA-IR values for insulin resistance and the Si-clamp values for insulin sensitivity were internally consistent and robust at demarcating IRO, IRN, ISO and ISN subjects (table 1). Using the BMI cut-off of $>30 \mathrm{~kg} / \mathrm{m}^{2}$ and defining prevalent insulin resistance as Si-clamp $<0.1 \mu \mathrm{mol} / \mathrm{kg}$ fat-free mass $/ \mathrm{min} / \mathrm{pmol} / \mathrm{L}$ and $/$ or HOMA-IR $>2.5,30 \%(96 / 320)$ of the study cohort (or $55.8 \%$ of the obese subjects) had ISO. In contrast, the IRN status was observed in only $5.3 \%(17 / 320)$ of the cohort (or $11.5 \%$ of non-obese subjects) (table 1). HOMA-IR data were obtained in all 320 subjects, of whom 206 underwent hyperinsulinemic euglycemic clamp to generate the Si-clamp data.

\section{Cardiometabolic measures}

Among the obese subgroups, ISO subjects had lower values for waist circumference $(p=0.007)$, total body fat $(\mathrm{p}=0.009)$, trunk fat $(\mathrm{p}=0.0032)$, serum triglycerides $(p=0.0019)$, hsCRP $(p=0.013)$ and insulin secretion $(\mathrm{p}=0.0061)$, and higher adiponectin levels $(\mathrm{p}=0.003)$ compared with IRO subjects (table 2). The ISO group also had lower values for basal (HOMA-B, $\mathrm{p}<0.0001$ ) and glucose-stimulated (AIR, $\mathrm{p}<0.0061)$ insulin secretion compared with the IRO group. Systolic and diastolic BP were not significantly different between the two obese subgroups. In the non-obese subgroups, IRN subjects had higher values for waist circumference $(\mathrm{p}<0.0001)$, systolic $(\mathrm{p}=0.03)$ and diastolic $(\mathrm{p}=0.009) \mathrm{BPs}$, 
Table 2 Cardiometabolic characteristics of study subjects by obesity and insulin sensitivity status

\begin{tabular}{lllllll}
\hline Characteristic & IRO & ISO & p Value & IRN & ISN & p Value \\
\hline Waist circumference $(\mathrm{cm})$ & $107 \pm 11.9$ & $102 \pm 11.7$ & 0.007 & $92.5 \pm 8.10$ & $81.7 \pm 9.84$ & $<0.0001$ \\
\hline Total fat $(\mathrm{kg})$ & $42.2 \pm 12.9$ & $37.6 \pm 10.6$ & 0.015 & $24.5 \pm 3.92$ & $20.3 \pm 6.10$ & 0.009 \\
\hline Trunk fat $(\mathrm{kg})$ & $21.6 \pm 6.49$ & $18.8 \pm 5.40$ & 0.0032 & $13.1 \pm 2.21$ & $9.19 \pm 3.12$ & $<0.0001$ \\
\hline SBP $(\mathrm{mm} \mathrm{Hg})$ & $127 \pm 16.3$ & $124 \pm 15.5$ & 0.16 & $123 \pm 18.3$ & $115 \pm 12.6$ & 0.03 \\
\hline DBP $(\mathrm{mm} \mathrm{Hg})$ & $75 \pm 8.86$ & $73 \pm 8.60$ & 0.19 & $78 \pm 9.67$ & $71 \pm 8.43$ & 0.009 \\
\hline HDL-C $(\mathrm{mg} / \mathrm{dl})$ & $48.8 \pm 12.0$ & $49.9 \pm 10.9$ & 0.54 & $44.8 \pm 9.89$ & $57.0 \pm 14.9$ & 0.0013 \\
\hline Triglycerides $(\mathrm{mg} / \mathrm{dl})$ & $115 \pm 57.7$ & $89.7 \pm 47.4$ & 0.0019 & $134 \pm 75.4$ & $83.0 \pm 48.8$ & 0.0003 \\
\hline Leptin $(\mathrm{ng} / \mathrm{ml})$ & $59.9 \pm 45.0$ & $48.1 \pm 36.1$ & 0.06 & $23.5 \pm 15.6$ & $15.5 \pm 13.0$ & 0.02 \\
\hline hsCRP $(\mathrm{mg} / \mathrm{L})$ & $6.34 \pm 6.71$ & $4.70 \pm 7.15$ & 0.013 & $3.06 \pm 3.37$ & $1.83 \pm 2.65$ & 0.08 \\
\hline Adiponectin $(\mu \mathrm{g} / \mathrm{mL})$ & $7.38 \pm 3.96$ & $9.34 \pm 4.46$ & 0.003 & $6.56 \pm 3.54$ & $10.8 \pm 6.02$ & 0.006 \\
\hline HOMA-B $(\%)$ & $158 \pm 62.1$ & $77.3 \pm 41.3$ & $<0.0001$ & $132 \pm 57.1$ & $62.4 \pm 62.7$ & $<0.0001$ \\
\hline AIR $(\mu \mathrm{U} / \mathrm{ml})$ & $119 \pm 91.9$ & $84.3 \pm 69.7$ & 0.0061 & $135 \pm 77.6$ & $62.2 \pm 52.7$ & $<0.0001$ \\
\hline REE $(\mathrm{kcal} / \mathrm{kgFFM})$ & $29.3 \pm 5.70$ & $29.2 \pm 6.20$ & 0.88 & $29.8 \pm 3.25$ & $30.2 \pm 5.42$ & 0.79 \\
\hline To & & & &
\end{tabular}

To convert the values for insulin to picomoles per liter, multiply by 7.175. To convert the values for HDL cholesterol to millimoles per liter, multiply by 0.02586 . To convert the values for triglycerides to millimoles per liter, multiply by 0.01129 .

AIR, acute insulin response to intravenous glucose; DBP, diastolic blood pressure; FFM, fat-free mass; HDL-C, high-density lipoprotein cholesterol; HOMA-B, homeostasis model of beta-cell function (\%); hsCRP, high-sensitivity $\mathrm{C}$ reactive protein; IRN, insulin-resistant nonobese; IRO, insulin-resistant obese; ISN, insulin-sensitive non-obese; ISO, insulin-sensitive obese; SBP, systolic blood pressure; REE, resting energy expenditure.

total body fat $(\mathrm{p}=0.015)$, trunk fat $(\mathrm{p}=0.0032)$, serum triglycerides $(p=0.0019)$ and leptin $(p=0.02)$, and lower adiponectin $(\mathrm{p}=0.006)$ and HDL cholesterol $(\mathrm{p}=0.0013)$ levels compared with ISN subjects. The IRN group also had higher values for basal (HOMA-B, $\mathrm{p}<0.0001)$ and glucose-stimulated (AIR, $\mathrm{p}<0.0001)$ insulin secretion compared with ISN lean subjects. Resting energy expenditure, normalized to lean mass, did not differ significantly across the four comparison groups. The cardiometabolic characteristics are summarized in table 2.

\section{Progression to pre-diabetes}

During 5.5 years of follow-up (mean 2.62 years), 100 of the 320 subjects developed incident pre-diabetes and 10 additional participants were diagnosed with T2D. Figure 2A shows the clamp-derived measures of insulin sensitivity in obese and non-obese participants. In the obese group, 37 of 76 IRO subjects (compared with 30 of 96 ISO subjects) developed pre-diabetes/T2D during follow-up. In the non-obese group, 8 of 17 IRN subjects (compared with 34 of 131 ISN subjects) developed incident pre-diabetes/ T2D. The cumulative incidence of pre-diabetes/T2DM was $48.7 \%$ in the IRO group, $31.3 \%$ in the ISO group, $47.1 \%$ in the IRN group and $26.0 \%$ in the ISN group $\left(\chi^{2}\right.$ for trend $\mathrm{p}=0.0024$ ) (figure $2 \mathrm{~B}$ ). Among the obese group, participants whose insulin sensitivity (Si-clamp) was above the 25th percentile had a $37 \%$ lower risk of incident pre-diabetes/T2D compared with those whose insulin sensitivity was below the 25 th percentile or whose insulin resistance (HOMA-IR) was above the 75th percentile. Among the non-obese participants, the presence of insulin resistance at baseline predicted a nominal $80 \%$ higher risk of incident dysglycemia compared with ISN subjects. The Kaplan-Meier disease-free survival analysis (figure 2C) showed a higher pre-diabetes/T2D survival probability for ISO subjects versus IRO subjects, and a lower survival probability for IRN subjects versus ISN participants $(\mathrm{p}=0.0001)$.

\section{DISCUSSION}

The existence of insulin-sensitive (metabolically 'healthy') and insulin-resistant, (metabolically 'unhealthy') phenotypes of obese and lean subjects has been the subject of several previous reports. ${ }^{1-10} 16$ However, there is a lack of consistency in the definition of those phenotypes, and their generalizability across racial/ethnic groups is unclear. Although insulin sensitivity is implied as a foundational construct in the classification of individuals into metabolically healthy versus unhealthy groups, it is seldom measured. ${ }^{1-1016}$ Of the studies that assessed insulin sensitivity, calculation of HOMA-IR has been the predominant approach, ${ }^{7} 1620$ although some recent studies have used the more rigorous hyperinsulinemic euglycemic clamp. ${ }^{17-20}$ As is well-known (and confirmed in the present study, figure 1), the correlation between HOMA-IR (an estimate of hepatic insulin sensitivity) and Si-clamp (a direct measurement of whole-body insulin sensitivity, using hyperinsulinemic euglycemic clamp) is far from perfect. ${ }^{32} 33$

Using a stricter definition of insulin sensitivity in the present study, we validate the existence of insulin-sensitive and insulin-resistant phenotypes among obese and non-obese individuals. Our study cohort had a large representation (55\%) of AA, which allowed us to specifically document the fidelity of these phenotypes in a 

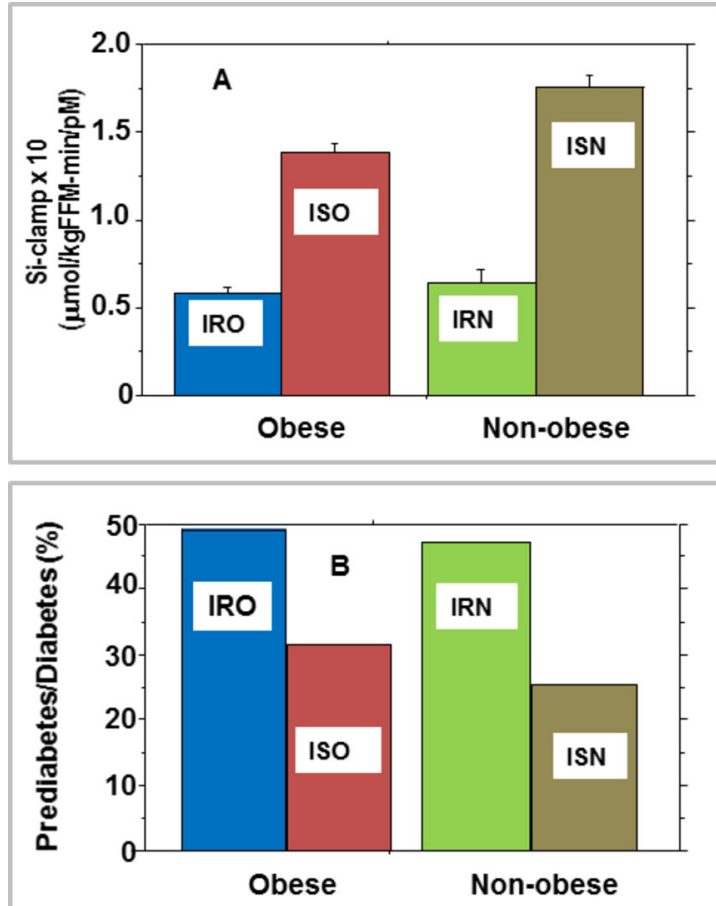

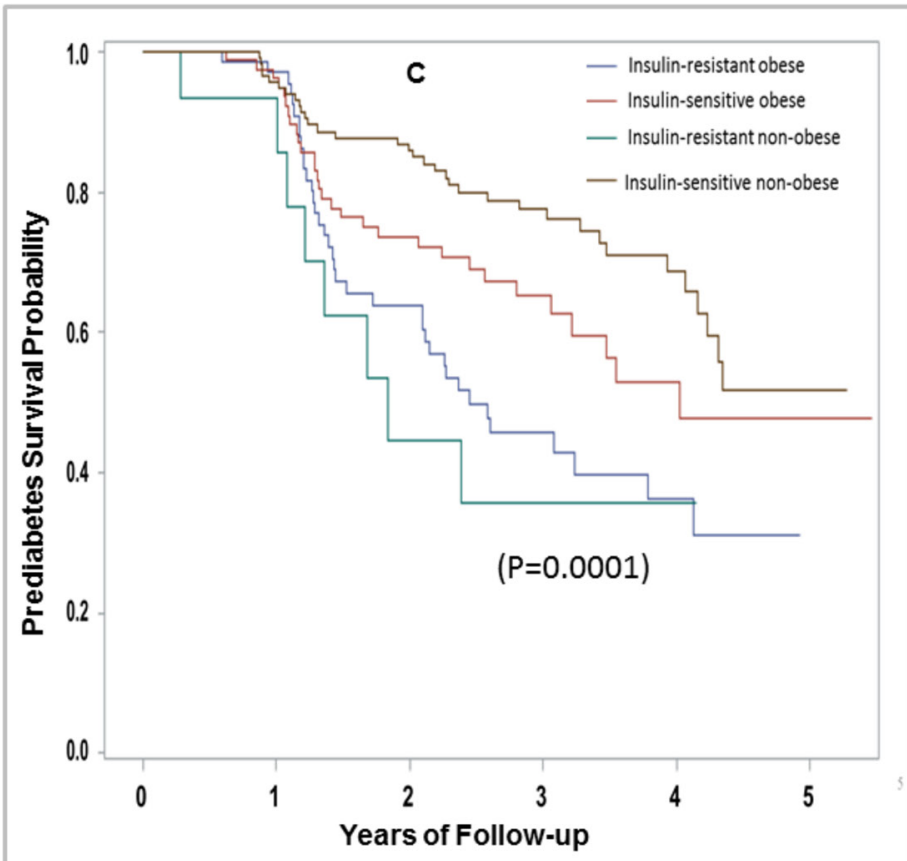

Figure 2 Insulin sensitivity (Si-clamp) in obese and non-obese study subjects (A) and cumulative incidence of pre-diabetes/ type 2 diabetes in insulin-resistant obese (IRO), insulin-sensitive obese (ISO), insulin-resistant non-obese (IRN) and insulinsensitive non-obese (ISN) subjects (B). The cumulative incidence was $48.7 \%$ in IRO, 30.9\% in ISO, $47.1 \%$ in IRN and $26 \%$ in ISN groups, respectively ( $\chi^{2} \mathrm{P}$ for trend=0.0024). (C) Kaplan-Meier plot of pre-diabetes/type 2 diabetes survival among participants stratified by IRO, IRN, ISO or ISN status (log-rank sum $\mathrm{p}=0.0001$ ).

non-Caucasian population. Unlike previous contributions on the subject, where the components of the metabolic syndrome were substituted as surrogates for insulin sensitivity, our approach stratified obese and non-obese study subjects purely based on insulin sensitivity. The threshold for insulin sensitivity used in the present study $(>0.1$ $\mu \mathrm{mol} / \mathrm{kg}$ fat-free mass $/ \mathrm{min} / \mathrm{pmol} / \mathrm{L}$ ) corresponds to a level previously characterized as indicative of insulin-sensitive, glucose-tolerant status among healthy subjects ${ }^{34}$; the HOMA-IR cut-off point for insulin resistance $(\geq 2.5)$ in the present study is also consistent with published practice. ${ }^{81620}$ We observed a plethora of cardiometabolic risk markers that aligned congruently with insulin sensitivity status, with high fidelity in obese and non-obese subjects. Several of these markers (including waist circumference, total and abdominal fat, hsCRP, adiponectin, hyperinsulinemia, BP, triglycerides, and HDL cholesterol) were differentially expressed among ISO, ISN, IRO and IRN participants in our biracial cohort. In general, the finding of preserved insulin sensitivity in obese individuals attenuated the expected adverse cardiometabolic profile, and insulin resistance in non-obese individuals was associated with an adverse cardiometabolic profile. It is noteworthy that these findings were observed in normoglycemic individuals, before any confounding effects of hyperglycemia-induced perturbations. ${ }^{22} 23$

Our findings support the existence of heterogeneity in cardiometabolic risk status among similarly obese or non-obese individuals. We show that measured insulin sensitivity correctly identified the expected pattern of co-segregation of cardiometabolic risk markers, regardless of obese or non-obese status. Although direct comparison is difficult, due to methodological differences, our ISO subjects display the properties associated with the MHO phenotype and our IRO subjects would be analogous to the 'metabolically unhealthy obese' (MUO) subjects described in the literature ${ }^{1-10}{ }^{16}$ Similarly, the non-obese subjects with insulin resistance in our study (IRN) resemble the MONW individuals and our ISN subjects are akin to the 'metabolically healthy, normal weight individuals' (MHNW). ${ }^{1-10}{ }^{16}$ Note, however, that our study population was a healthy cohort with a mean $\mathrm{BP}$ of $<130 / 80 \mathrm{~mm} \mathrm{Hg}$, triglycerides of $<150 \mathrm{mg} / \mathrm{dL}$, FPG of $<100 \mathrm{mg} / \mathrm{dL}$, HDL cholesterol of $>50 \mathrm{mg} / \mathrm{dL}$ and waist circumference of $97.9 \mathrm{~cm}$ in men and $92.7 \mathrm{~cm}$ in women. The traditional approach of classifying $\mathrm{MHO} / \mathrm{MUH}$ and MONW/MHNW phenotypes, based on the presence or absence of two of five metabolic syndrome markers, would have misclassified nearly all the IRO and IRN subjects as 'metabolically healthy'. ${ }^{2-11} 16$

In contrast, our approach of phenotyping obese individuals as ISO or IRO and non-obese subjects as IRN or ISN, based on measured insulin sensitivity, was remarkably sensitive at identifying a clustering of subclinical perturbations in cardiometabolic risk factors (table 2). Indeed, ours is the first study that has employed rigorous 
measurement of insulin sensitivity to document healthy and unhealthy phenotypes of obesity and leanness in a prospective biracial cohort. The frequency of the MHO phenotype was $30 \%$ in our study population $(55.8 \%$ of the obese group). Previous estimates of the MHO phenotype from mostly Caucasian populations have been in the $10 \%-40 \% .{ }^{1-10} 1635$ In a study of 343 AA obese subjects, $96(28 \%)$ were classified as having the MHO phenotype, based on components of the metabolic syndrome. ${ }^{36}$ In another report, 36 of 126 obese AA $(28.5 \%)$ had the MHO phenotype, defined as a BMI $\geq 30 \mathrm{~kg} / \mathrm{m} 2$, HDL cholesterol $\geq 40 \mathrm{mg} / \mathrm{dL}$, and absence of T2D or hypertension. $^{37}$

The frequency of the ISO phenotype in the obese group of our study $(55.8 \%)$ is numerically higher than previously reported estimates for MHO. Data from National Health and Nutrition Examination Survey 1999-2004 showed that $51.3 \%$ of overweight adults and $31.7 \%$ of obese adults were metabolically healthy. ${ }^{9}$ Besides methodological differences, the reason for the higher frequency of the ISO phenotype might be the unique characteristics of our study subjects, all of whom have parents with T2DM but were required to have normoglycemia at enrollment. Given their mean age of 44 years and their strong genetic risk for T2DM, it is likely that obese offspring of parents with T2DM who met the eligibility criterion of normoglycemia had an enrichment of subjects with healthier insulin sensitivity. Thus, a more representation cross-section of the general population would be expected to have a lower proportion of individuals with ISO than was observed in our study. We also observed that the IRN phenotype was present in 5\% of our entire cohort (11.5\% of non-obese subjects). Other studies have reported frequencies ranging from $5 \%$ to $45 \%$ for the MONW. ${ }^{9101635}$ Based on our population distributions, the ISO (or MHO-like) phenotype is more prevalent than the IRN (or MONW-like) phenotype, which would be teleologically congruent. Preservation of insulin sensitivity would be an adaptive trait among obese persons, whereas an insulin-resistant lean phenotype would seem maladaptive.

The insulin-sensitive or MHO phenotype has been associated with decreased risk of cardiovascular disease, ${ }^{16} 2038$ although mortality data are discordant. ${ }^{39}$ In the predominantly Caucasian Framingham Offspring Study, lower insulin resistance (assessed by HOMA-IR) was associated with decreased risk of incident T2DM among obese subjects, whereas higher insulin resistance predicted increased diabetes risk in non-obese subjects. ${ }^{20}$ In the present study, ISO (compared with IRO) subjects had a $37 \%$ relative decrease, and IRN (compared with ISN) subjects had an $80 \%$ relative increase, in incident pre-diabetes/T2D. Although the small number of participants in the IRN group should temper conclusions, our findings extend the previous report from the Framingham Offspring Study ${ }^{20}$ to a broader demographic group and a more proximal stage in the pathogenesis of diabetes. The mechanisms underlying the existence of ISO, ISN,
IRO and IRN phenotypes are not fully understood. Putative mechanisms/mediators include visceral fat abundance and hepatic enzymatic activity, ${ }^{840}$ pathways involving natural killer cells ${ }^{41}$ and endocannabinoids, ${ }^{42}$ and expression of lipogenic transcription factors, ${ }^{43}$ among others. In experimental models, overexpression of adiponectin recapitulates elements of the MHO phenotype. ${ }^{44}$ In the present study, the insulin-sensitive phenotype was associated with higher adiponectin levels compared with the insulin-resistant phenotype in obese and non-obese subjects. Previous reports have also associated higher adiponectin levels with MHO phenotype ${ }^{3645}$ and decreased risk of incident pre-diabetes. ${ }^{46}$

The strengths of our analysis include the unique biracial cohort, specific documentation of insulin sensitivity, and the longitudinal follow-up design. Furthermore, by studying a generally healthy, normoglycemic cohort, we avoided confounding from secondary effects of hyperglycemia, dyslipidemia or other perturbations on important cardiometabolic variables. We also obtained behavioral measures (food habits and physical activity), which, though self-reported, nonetheless allowed us to eliminate gross differences as a possible explanation for our findings. The major weakness is that our study was confined to offspring of parents with T2D, and thus our findings may not be generalizable to the larger population. Also, the hyperinsulinemic euglycemic clamp is tedious and not amenable to translation. Indeed, even the simpler HOMA-IR, which requires measurement of fasting plasma insulin and glucose, may be beyond the reach of primary care practice. It is, therefore, reassuring that our study essentially validates the existence of metabolically healthy and unhealthy obese and non-obese phenotypes that were largely deduced from the use of simpler clinical traits and routine laboratory tests. Also, pre-diabetes is a heterogenous condition, and individuals with impaired fasting glucose and those with impaired glucose tolerance may not have identical penetrance of insulin resistance and related metabolic perturbations. ${ }^{47}$ Furthermore, the MHO phenotype may not be stable over time, as nearly one-third of individuals have been reported to convert to a metabolically less healthy phenotype. $^{48}$

In conclusion, using rigorous methodology for quantitation of insulin sensitivity, we have demonstrated that ISO is quite prevalent, is associated with a favorable cardiometabolic profile, and is protective of incident pre-diabetes/T2D among AA and EA with parental history of diabetes.

Acknowledgements We are indebted to the participants who volunteered for this study.

Collaborators POP-ABC Research Group: Current: Samuel Dagogo-Jack, MD (Principal Investigator), Ann Ammons, BS, Amy Brewer, MS, RD, Fatoumatta Ceesay, BS, Ibiye Owei, MBBS, MPH, Casey Provo, MS, LDN, Nkiru Umekwe, MBBS, Jim Wan, PhD. Past members: Emmanuel Chapp-Jumbo, MBBS (2009-2011), Chimaroke Edeoga, MBBS, MPH (2007-2013), Ruben Cuervo, MD (2006-2007), Sotonte Ebenibo, MBBS, MPH (2011-2014), Nonso Egbuonu, MBBS (2007-2010), Nicoleta lonica, MD (2007-2008), Dorota Malinowski, MD (2007-2008). Consultant: Steven Haffner, MD; Data and Safety Officer: Murray Heimberg, MD, PhD. 
Contributors SD-J: principal investigator, designed study, wrote manuscript. I0: collected data, reviewed and revised manuscript. NU: collected data, reviewed and revised manuscript. CP: collected data, reviewed and revised manuscript. JW: performed statistical analysis, reviewed and revised manuscript. SD-J, as the guarantor, takes full responsibility for the work including the study design, access to data, and the decision to submit and publish the manuscript.

Funding The POP-ABC study was supported by grants R01 DK067269 and R01 DK067269-04S1 from the National Institutes of Health and grant 7-07-MN-13 from the American Diabetes Association. The funding sources had no role in the design and execution of the POP-ABC study, or analysis and publication of the data obtained from the study.

\section{Competing interests None declared.}

Ethics approval The University of Tennessee Institutional Review Board. Provenance and peer review Not commissioned; internally peer reviewed. Data sharing statement № additional data are available.

Open Access This is an Open Access article distributed in accordance with the Creative Commons Attribution Non Commercial (CC BY-NC 4.0) license, which permits others to distribute, remix, adapt, build upon this work non-commercially, and license their derivative works on different terms, provided the original work is properly cited and the use is non-commercial. See: http://creativecommons.org/ licenses/by-nc/4.0/

(c) Article author(s) (or their employer(s) unless otherwise stated in the text of the article) 2017. All rights reserved. No commercial use is permitted unless otherwise expressly granted.

\section{REFERENCES}

1. Ferrannini E, Natali A, Bell P, et al. Insulin resistance and hypersecretion in obesity. European Group for the Study of Insulin Resistance (EGIR). J Clin Invest 1997;100:1166-73.

2. Brochu M, Tchernof A, Dionne IJ, et al. What are the physical characteristics associated with a normal metabolic profile despite a high level of obesity in postmenopausal women? J Clin Endocrinol Metab 2001;86:1020-5.

3. Stefan N, Kantartzis K, Machann J, et al. Identification and characterization of metabolically benign obesity in humans. Arch Intern Med 2008;168:1609-16.

4. Pajunen P, Kotronen A, Korpi-Hyövälti E, et al. Metabolically healthy and unhealthy obesity phenotypes in the general population: the FIN-D2D Survey. BMC Public Health 2011;11:754.

5. Blüher M. The distinction of metabolically 'healthy' from 'unhealthy' obese individuals. Curr Opin Lipidol 2010;21:38-43.

6. Calori G, Lattuada G, Piemonti L, et al. Prevalence, metabolic features, and prognosis of metabolically healthy obese Italian individuals: the Cremona Study. Diabetes Care 2011;34:210-5.

7. Soverini V, Moscatiello S, Villanova N, et al. Metabolic syndrome and insulin resistance in subjects with morbid obesity. Obes Surg 2010;20:295-301.

8. Conus F, Rabasa-Lhoret R, Péronnet F. Characteristics of metabolically obese normal-weight (MONW) subjects. Appl Physiol Nutr Metab 2007;32:4-12.

9. Wildman RP, Muntner P, Reynolds K, et al. The obese without cardiometabolic risk factor clustering and the normal weight with cardiometabolic risk factor clustering: prevalence and correlates of 2 phenotypes among the US population (NHANES 1999-2004). Arch Intern Med 2008;168:1617-24.

10. Ding C, Chan Z, Magkos F. Lean, but not healthy: the 'metabolically obese, normal-weight' phenotype. Curr Opin Clin Nutr Metab Care 2016;19:408-17.

11. National Cholesterol Education Program (NCEP) Expert Panel on Detection, Evaluation, and Treatment of High Blood Cholesterol in Adults (Adult Treatment Panel III). Third Report of the National Cholesterol Education Program (NCEP) Expert Panel on Detection, Evaluation, and Treatment of High Blood Cholesterol in Adults (Adult Treatment Panel III) final report. Circulation 2002;106:3143-421.

12. Taggart H, Stout RW. Reduced high density lipoprotein in stroke: relationship with elevated triglyceride and hypertension. Eur J Clin Invest 1979;9:219-21.

13. Albrink MJ, Krauss RM, Lindgrem FT, et al. Intercorrelations among plasma high density lipoprotein, obesity and triglycerides in a normal population. Lipids 1980;15:668-76.

14. Sumner AE. Ethnic differences in triglyceride levels and high-density lipoprotein lead to underdiagnosis of the metabolic syndrome in black children and adults. J Pediatr 2009;155:e7-e11:S7.
15. Laws A, Reaven GM. Evidence for an independent relationship between insulin resistance and fasting plasma HDLcholesterol, triglyceride and insulin concentrations. J Intern Med 1992;231:25-30.

16. Roberson LL, Aneni EC, Maziak W, et al. Beyond BMI: The "Metabolically healthy obese" phenotype \& its association with clinical/subclinical cardiovascular disease and all-cause mortality -a systematic review. BMC Public Health 2014;14:14.

17. Karelis AD, Faraj M, Bastard JP, et al. The metabolically healthy but obese individual presents a favorable inflammation profile. J Clin Endocrinol Metab 2005;90:4145-50.

18. Marini MA, Frontoni S, Succurro E, et al. Differences in insulin clearance between metabolically healthy and unhealthy obese subjects. Acta Diabetol 2014;51:257-61.

19. ter Horst KW, Gilijamse PW, Koopman KE, et al. Insulin resistance in obesity can be reliably identified from fasting plasma insulin. Int $J$ Obes 2015;39:1703-9.

20. Meigs JB, Wilson PW, Fox CS, et al. Body mass index, metabolic syndrome, and risk of type 2 diabetes or cardiovascular disease. $J$ Clin Endocrinol Metab 2006;91:2906-12.

21. Bell JA, Kivimaki M, Hamer M, et al. Metabolically healthy obesity and risk of incident type 2 diabetes: a meta-analysis of prospective cohort studies. Obes Rev 2014;15:504-15.

22. Ganda OP, Soeldner JS, Gleason RE, et al. Alterations in plasma lipids in the presence of mild glucose intolerance in the offspring of two type II diabetic parents. Diabetes Care 1985;8:254-60.

23. Stentz FB, Kitabchi AE. Hyperglycemia-induced activation of human T-lymphocytes with de novo emergence of insulin receptors and generation of reactive oxygen species. Biochem Biophys Res Commun 2005;335:491-5.

24. Dagogo-Jack S, Edeoga C, Nyenwe E, et al. Pathobiology of Prediabetes in a Biracial Cohort (POP-ABC): design and methods. Ethn Dis 2011;21:33-9.

25. Ebenibo S, Edeoga C, Ammons A, et al. Pathobiology of Prediabetes in a Biracial Cohort (POP-ABC) Research Group. Recruitment strategies and yields for the Pathobiology of Prediabetes in a Biracial Cohort: a prospective natural history study of incident dysglycemia. BMC Med Res Methodol 2013;13:64.

26. Dagogo-Jack S, Edeoga C, Ebenibo S, et al. Pathobiology of Prediabetes in a Biracial Cohort (POP-ABC) Research Group. pathobiology of Prediabetes in a Biracial Cohort (POP-ABC) study: baseline characteristics of enrolled subjects. J Clin Endocrinol Metab 2013;98:120-8.

27. Boucher AB, Adesanya EA, Owei I, et al. Dietary habits and leisuretime physical activity in relation to adiposity, dyslipidemia, and incident dysglycemia in the pathobiology of prediabetes in a biracial cohort study. Metabolism 2015;64:1060-7.

28. Genuth S, Alberti KG, Bennett P, et al. The expert committee on the diagnosis and classification of Diabetes Mellitus: 2003 followup report on the diagnosis of diabetes mellitus. Diabetes Care 2003;26:3160-7.

29. Dagogo-Jack S, Edeoga C, Ebenibo S, et al. Pathobiology of Prediabetes in a Biracial Cohort (POP-ABC) Research Group. lack of racial disparity in incident prediabetes and glycemic progression among black and white offspring of parents with type 2 Diabetes: the pathobiology of Prediabetes in a Biracial Cohort (POP-ABC) Study. J Clin Endocrinol Metab 2014;99:E1078-87.

30. DeFronzo RA, Tobin JD, Andres R, et al. Glucose clamp technique: a method for quantifying insulin secretion and resistance. Am J Physiol 1979;237:E214-22.

31. Matthews DR, Hosker JP, Rudenski AS, et al. Homeostasis model assessment: insulin resistance and beta-cell function from fasting plasma glucose and insulin concentrations in man. Diabetologia 1985;28:412-9.

32. Ferrara CM, Goldberg AP. Limited value of the homeostasis model assessment to predict insulin resistance in older men with impaired glucose tolerance. Diabetes Care 2001;24:245-9.

33. Otten J, Ahrén B, Olsson T, et al. Surrogate measures of insulin sensitivity vs the hyperinsulinaemic-euglycaemic clamp: a metaanalysis. Diabetologia 2014;57:1781-8.

34. Dagogo-Jack S, Askari H, Tykodi G, et al. Glucoregulatory physiology in subjects with low-normal, high-normal, or impaired fasting glucose. J Clin Endocrinol Metab 2009;94:2031-6.

35. Rotar O, Boyarinova M, Orlov A, et al. Metabolically healthy obese and metabolically unhealthy non-obese phenotypes in a russian population. Eur J Epidemiol 2017;32.

36. Doumatey AP, Bentley AR, Zhou J, et al. Paradoxical Hyperadiponectinemia is Associated With the Metabolically Healthy Obese (MHO) Phenotype in African Americans. J Endocrinol Metab 2012;2:51-65. 
37. Cherqaoui R, Kassim TA, Kwagyan J, et al. The Metabolically Healthy But Obese Phenotype in African Americans. J Clin Hypertens 2012;14:92-6.

38. Ogorodnikova AD, Kim M, McGinn AP, et al. Incident cardiovascular disease events in metabolically benign obese individuals. Obesity 2012;20:651-9.

39. Kuk JL, Ardern Cl. Are metabolically normal but obese individuals at lower risk for all-cause mortality? Diabetes Care 2009;32:2297-9.

40. Messier V, Karelis AD, Robillard ME, et al. Metabolically healthy but obese individuals: relationship with hepatic enzymes. Metabolism 2010;59:20-4.

41. Lynch LA, O'Connell JM, Kwasnik AK, et al. Are natural killer cells protecting the metabolically healthy obese patient? Obesity 2009;17:601-5.

42. Osei-Hyiaman D, DePetrillo M, Pacher P, et al. Endocannabinoid activation at hepatic $\mathrm{CB} 1$ receptors stimulates fatty acid synthesis and contributes to diet-induced obesity. J Clin Invest 2005;115:1298-305.

43. Kantartzis K, Fritsche A, Machicao F, et al. Upstream transcription factor 1 gene polymorphisms are associated with high antilipolytic insulin sensitivity and show gene-gene interactions. $J \mathrm{Mol}$ Med 2007;85:55-61.

44. Liu M, Xiang R, Wilk SA, et al. Fat-specific DsbA-L overexpression promotes adiponectin multimerization and protects mice from diet-induced obesity and insulin resistance. Diabetes 2012;61:2776-86.

45. Aguilar-Salinas CA, García EG, Robles L, et al. High adiponectin concentrations are associated with the metabolically healthy obese phenotype. J Clin Endocrinol Metab 2008;93:4075-9.

46. Jiang Y, Owei I, Wan J, et al. Adiponectin levels predict prediabetes risk: the Pathobiology of Prediabetes in A Biracial Cohort (POP-ABC) study. BMJ Open Diabetes Res Care 2016; 4:e000194.

47. Stefan N, Fritsche A, Schick F, et al. Phenotypes of prediabetes and stratification of cardiometabolic risk. Lancet Diabetes Endocrinol 2016;4:789-98.

48. Appleton SL, Seaborn CJ, Visvanathan R, et al. North West Adelaide Health Study Team. Diabetes and cardiovascular disease outcomes in the metabolically healthy obese phenotype: a cohort study. Diabetes Care 2013;36:2388-94. 